\title{
Rapid Parameterization Schemes for Aircraft Shape Optimization
}

\author{
$\mathrm{Wu} \mathrm{Li}{ }^{*}$ \\ NASA Langley Research Center, Hampton, Virginia 23681, USA
}

\begin{abstract}
A rapid shape parameterization tool called PROTEUS is developed for aircraft shape optimization. This tool can be applied directly to any aircraft geometry that has been defined in PLOT3D format, with the restriction that each aircraft component must be defined by only one data block. PROTEUS has eight types of parameterization schemes: planform, wing surface, twist, body surface, body scaling, body camber line, shifting/scaling, and linear morphing. These parametric schemes can be applied to two types of components: wing-type surfaces (e.g., wing, canard, horizontal tail, vertical tail, and pylon) and body-type surfaces (e.g., fuselage, pod, and nacelle). These schemes permit the easy setup of commonly used shape modification methods, and each customized parametric scheme can be applied to the same type of component for any configuration. This paper explains the mathematics for these parametric schemes and uses two supersonic configurations to demonstrate the application of these schemes.
\end{abstract}

\section{Nomenclature}

$c \quad=$ index for control point

$D=$ point in two-dimensional space

$f, g \quad=$ generic functions

$i, j \quad=\quad$ indices for points or parameters

$k=$ number of parameter locations

$m, n \quad=$ dimensions for data points

$P \quad=$ point in space

$Q \quad=\quad$ estimated unit normal vector of surface

$R \quad=\quad$ design variable for radial change

$S=$ design variable for scaling

$u, v \quad=$ independent variables of functions that define surfaces or curves

$W \quad=$ centroid of cross section

$x, y, z=$ the coordinates of a point in space

$\Delta Z \quad=$ design variable for change in $z$ coordinate

$\lambda, \mu \quad=$ parameters used to define locations of design variables or control points

\section{Introduction}

$\mathrm{P}$ ARAMETERIZATION methods are important for aircraft shape optimization. Samareh [1] presents an extensive survey of shape design and modification methods, including the following approaches: basic vector, domainelement, partial differential equation, discrete, polynomial and spline, computer-aided-design-(CAD)-based, analytical, and free-form deformation. The free-form deformation approach has been implemented in both a NASA in-house software code called MASSOUD [2] and a commercial software application called SCULPTOR ${ }^{\mathrm{TM}}$ [3]. These tools are used primarily for the modification of an existing computational fluid dynamics (CFD) geometry model. Recently, a new domain-element approach has been developed that uses radial basis functions to deform the geometry shape and the computational domain [4]. To generate a new aircraft geometry model, one could use either commercial CAD software or some customized parametric schemes. Vehicle Sketch Pad (VSP) [5] is a parametric aircraft shape design tool that uses polynomials and splines to define parametric shapes. VSP has an easy-to-use

*Senior Research Engineer, Aeronautics Systems Analysis Branch, Mail Stop 442. 
graphical interface for aircraft component design at the conceptual level and aims at the simple construction of aircraft components, such as a fuselage and wing, using only a few design variables. Another method for aircraftcomponent shape design uses the class and shape function transformation (CST) for geometry representation [6]. The CST method also permits the easy construction of aircraft components by representing each aircraft component as either a wing-airfoil type of surface or a body-cross-section type of surface. In reference [6], examples are given to demonstrate how wings, fuselages, and nacelles can be constructed by using specific shape functions for airfoils and body cross sections, respectively. Note the similarity between the CST and VSP parameterization schemes: in both methods, aircraft components are modeled as either wing-type or body-type surfaces. The simplicity of the component shape types allows the automated conversion of compatible aircraft components, which are defined by grid points, to parametric VSP geometry [7].

In practical applications, shape optimization usually is used to find desirable changes in the geometry that improve a baseline configuration in terms of some numerical objectives. Trial-and-error methods are necessary in the search for an effective shape parameterization scheme that yields a desirable optimal solution. As a result, there is a need for shape parameterization schemes that can be set up and modified easily. With the successful integration of automated CFD analysis into the early conceptual aircraft design phase [8,9], the same parameterization tool must be able to provide both the drastic shape changes that can occur during the concept exploration phase as well as the fine shape modifications that are necessary during the high-fidelity shape-tailoring stage. Parametric shape design tools such as MASSOUD and SCULPTOR are effective for CFD-based shape optimization of an existing CFD geometry model, but setting up a parametric scheme that reflects intuitive design modifications, such as changing the camber surface of a wing or the width distribution of a fuselage, can be quite time consuming. Further, modifying the intersection of two components can be extremely difficult. On the other hand, a parametric aircraft shape design tool such as VSP can be used for CFD-based shape design optimization once an automated CFD analysis is feasible [10]. Two reasons necessitate the development of a parameterization tool other than VSP for CFD-based shape optimization at the conceptual design level: (1) the discrepancy that occurs in the geometry when a third-party geometry is converted to a VSP parametric format (either manually or with the use of a conversion code) and (2) the unintended changes in geometry when a different parametric scheme is used for shape optimization.

In this paper, a rapid shape parameterization tool called PROTEUS is introduced for aircraft shape optimization. This parameterization tool provides eight schemes for body-type components (i.e., fuselage, nacelle, and pod) and wing-type components (i.e., wing, canard, horizontal tail, vertical tail, and pylon). This tool can be used to modify the shape of body-type and wing-type components for any given geometry in PLOT3D format. Each parameterization scheme for a given geometry model can be set up by (1) typing in the component name, (2) selecting a parametric scheme, (3) choosing the ranges for $u$ and $v$ that are affected by the parametric scheme (assuming that the component is implicitly defined by a three-dimensional surface on $(u, v)$ space), and (4) specifying the number of design variables in both the $u$ and $v$ directions and the corresponding $u$ and $v$ locations for the design variables.

The technical details of the parametric schemes are given in section II, while section III shows the application of these parametric schemes to both a VSP model of a supersonic concept and a Boeing concept of a low-boom supersonic transport. Concluding remarks are given in section IV.

\section{Parametric Schemes}

The parameterization tool PROTEUS has been implemented in ModelCenter ${ }^{\mathrm{TM}}$ [11] (see Fig. 1), but it can be used as a standalone code (with the manual setup of input parameters). The current version of PROTEUS provides the following eight parameterization schemes: planform, lifting surface, twist, body surface, body scaling, body camber line, shifting and scaling, and linear morphing. These schemes can be applied to any given geometry that is defined in either PLOT3D or Hermite format. Hermite format is similar to PLOT3D format but includes additional information about each component, such as the component name and type.

In Fig. 1, three parametric schemes are created for the input geometry in Hermite format: a planform scheme for the wing, a lifting-surface scheme for the horizontal tail, and a body-surface scheme for the interior surface of a nacelle. A user has the option to deactivate a parametric scheme by selecting the mode "None." In Fig. 1, the bodysurface scheme for the interior nacelle surface is not active, so the input geometry is modified only by the two active parametric schemes. These parametric schemes are applied sequentially to the input geometry, and two or more different parametric schemes of the same type can be used as necessary. After the parametric schemes have been defined, a user can run ModelCenter and view both the original and modified geometry shapes either within ModelCenter (which has a geometry viewer) or by using a visualization tool such as Tecplot 360 [12]. 


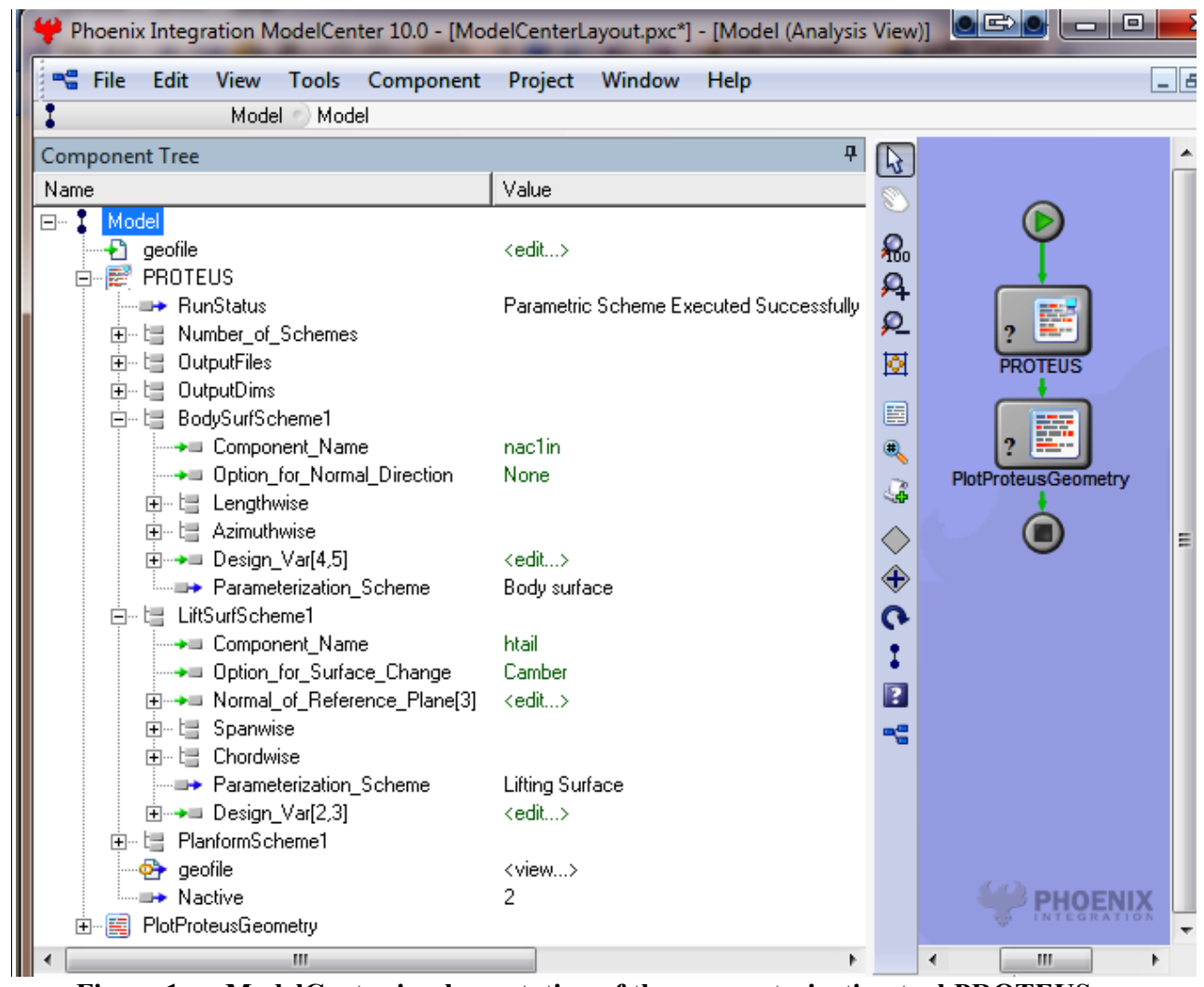

Figure 1. ModelCenter implementation of the parameterization tool PROTEUS.

PROTEUS assumes that a wing-type surface has a natural partition between the upper and lower surfaces, with an equal number of data points on both the upper and the lower surface for each airfoil section. Users can specify a normal direction vector of the plane for the planform, which is $(0,0,1)$ for a wing and $(0,1,0)$ for a vertical tail. With this assumption, the leading and trailing edges of a wing-type surface can easily be located. Suppose that the original wing-type component is defined by the data points:

$$
P[i, j]=(x[i, j], y[i, j], z[i, j]) \text { for } 1 \leq i \leq m \text { and } 1 \leq j \leq 2 n-1 \text {, }
$$

where each index $i$ corresponds to one airfoil section and the leading edge is defined by points for which $j=n$. Then, PROTEUS finds the best estimate $u_{i}$ of the percentage of the span location for the airfoil section $i$ and the best estimate $v_{j}$ of the percentage of the chord location for the data points $\{P[i, j], P[i, 2 \mathrm{n}-j]: 1 \leq i \leq m\}$. That is, $u_{i}$ and $v_{j}$ represent the best estimates of the percentage of the span location and the percentage of the chord location for both $P[i, j]$ and $P[i, 2 \mathrm{n}-j]$. Obviously, $u_{1}=v_{1}=0$ and $u_{m}=v_{n}=1$.

For the planform scheme, the planform shape of a wing-like surface can be changed by (1) specifying the design ranges for the leading and trailing edges:

$$
0 \leq u_{\min , \mathrm{le}}<u_{\max , \mathrm{le}} \leq 1 \text { and } 0 \leq u_{\min , \mathrm{te}}<u_{\max , \mathrm{te}} \leq 1
$$

and (2) selecting the locations of the control points:

$$
0 \leq \lambda_{1, \mathrm{le}}<\ldots<\lambda_{k 1, \mathrm{le}} \leq 1 \text { and } 0 \leq \lambda_{1, \mathrm{te}}<\ldots<\lambda_{k 2, \mathrm{te}} \leq 1
$$

In Fig. $2, u_{\min , \mathrm{le}}=0.3$ and $u_{\max , \mathrm{le}}=0.85$. The locations of the control points are relative to the design ranges. In Fig. $2, k_{1}=4, \lambda_{1, \mathrm{le}}=0.3, \lambda_{2, \mathrm{le}}=0.4, \lambda_{3, \mathrm{le}}=0.6$, and $\lambda_{4, \mathrm{le}}=0.8$. The actual locations of the control points for the leading edge are $u_{i, \mathrm{le}}=u_{\text {min,le }}+\lambda_{i, \mathrm{le}} \cdot\left(u_{\mathrm{max}, \mathrm{le}}-u_{\mathrm{min}, \mathrm{le}}\right)$. The relative location $\lambda_{1, \mathrm{le}}$ must be greater than 0 if $u_{\mathrm{min}, \mathrm{le}}>0$

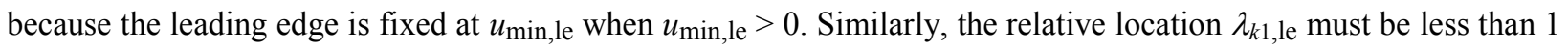


if $u_{\text {max,le }}<1$. The leading edge modification curve $f(u)$ is either a piecewise linear curve or a B-spline curve interpolation of the control points

$$
\left(D_{1}, \ldots, D_{k 1}\right): f\left(u_{i, \mathrm{le}}\right)=\left(f_{1}\left(u_{i, \mathrm{le}}\right), f_{2}\left(u_{i, \mathrm{le}}\right)\right)=D_{i} \text { for } 1 \leq i \leq k_{1} .
$$

In Fig. $2, u_{\text {min,te }}=0, u_{\text {max,te }}=0.9, k_{2}=3, \lambda_{1, \text { te }}=0, \lambda_{2, \text { te }}=0.4$, and $\lambda_{3, \text { te }}=0.8$. Note that each control point $D_{i}$ has two design variables that control the change of the planform in the span and chord directions. For simplicity, the formula for defining the modified leading edge of a standard wing is

$$
x_{\text {new }}[i, n]=x[i, n]+f_{1}\left(u_{i}\right) \text { and } y_{\text {new }}[i, n]=y[i, n]+f_{2}\left(u_{i}\right) \text { for } u_{\text {min,le }} \leq u_{i} \leq u_{\text {max }, \mathrm{le}}
$$

If $f(u)$ is a B-spline curve and $u_{\text {min,le }}>0$, then the first derivative $f^{\prime}\left(u_{\min , \mathrm{le}}\right)$ is equal to $(0,0)$ to ensure a smooth blending of the modified and unmodified parts of the leading edge. The trailing modification is implemented similarly by using a trailing-edge modification curve $g(u)$. Then, the wing surface points are modified by using a linear deformation:

$$
x_{\text {new }}[i, j]=x[i, j]+\left(1-v_{j}\right) \cdot f_{1}\left(u_{i}\right)+v_{j} \cdot g_{1}\left(u_{i}\right) \text { and } y_{\text {new }}[i, j]=y[i, j]+\left(1-v_{j}\right) \cdot f_{2}\left(u_{i}\right)+v_{j} \cdot g_{2}\left(u_{i}\right) .
$$

The modified wing in Fig. 2 is obtained by changing the $x$ coordinates for the seven control points.

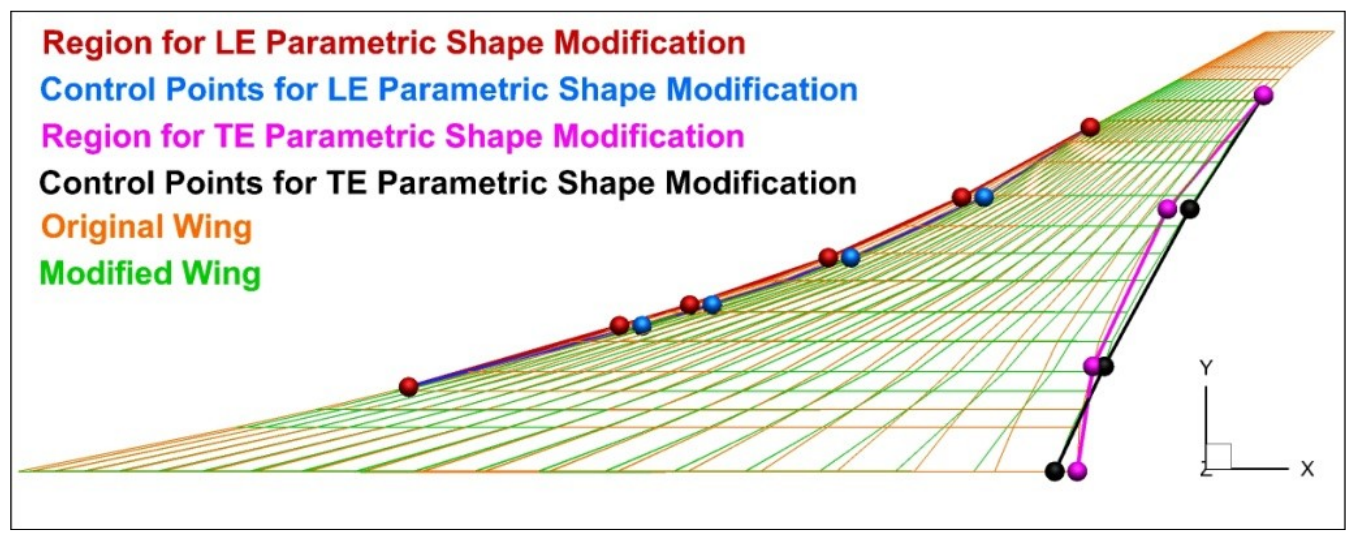

Figure 2. A parametric scheme for wing planform modification.

Note that the locations of the control points are arbitrary and different locations lead to different parametric schemes. Moreover, by simply changing the design ranges that are defined by $u_{\min , l e}, u_{\max , l e}, u_{\min , \text { te }}$, and $u_{\max , \text { te }}$, a user can either zoom in to change a smaller region of the planform or zoom out to change a larger region of the planform. Also, locations of the control points can be added or removed to increase or decrease the resolution of the design space because the number of design variables is $2 \cdot\left(k_{1}+k_{2}\right)$. This decoupling of the design space and the geometry definitions is quite useful in the search for an effective parametric scheme for shape optimization. This is a common feature for all parametric schemes in PROTEUS.

For the lifting-surface scheme, the camber or thickness of a wing-like surface can be changed by (1) specifying the design ranges for the span and chord directions

$$
0 \leq u_{\min }<u_{\max } \leq 1 \text { and } 0 \leq v_{\min }<v_{\max } \leq 1
$$

and (2) selecting the locations of the control points:

$$
0 \leq \lambda_{1}<\ldots<\lambda_{k 1} \leq 1 \text { and } 0 \leq \mu_{1}<\ldots<\mu_{k 2} \leq 1
$$

The number of design variables is $k_{1} \cdot k_{2}$. The actual locations of the control points are $u_{i, c}=u_{\min }+\lambda_{i} \cdot\left(u_{\max }-u_{\min }\right)$ and $v_{j, c}=v_{\min }+\mu_{j} \cdot\left(v_{\max }-v_{\min }\right)$. Again, the control surface $f(u, v)$ is defined by a B-spline interpolation of the control points 


$$
\left\{\Delta Z_{i, j}: 1 \leq i \leq k_{1} \text { and } 1 \leq j \leq k_{2}\right\}: f\left(u_{i, c}, v_{j, c}\right)=\Delta Z_{i, j}
$$

Here, $\Delta Z_{i, j}$ is the change to the camber or thickness at the location $\left(u_{i, c}, v_{j, c}\right)$. Then, the wing surface points are modified by adding $f\left(u_{i}, v_{j}\right)$ to $z[i, j]$ and $z[i, 2 \mathrm{n}-j]$ to change the camber. A change in the thickness is implemented by adding $f\left(u_{i}, v_{j}\right) / 2$ to $z[i, j]$ and subtracting $f\left(u_{i}, v_{j}\right) / 2$ from $z[i, 2 \mathrm{n}-j]$.

Figure 3 illustrates a parametric scheme for modifying the wing camber or thickness with three design variables. In this example, $u_{\min }=0.2, u_{\max }=0.7, v_{\min }=0.3, v_{\max }=0.6, k_{1}=3, \lambda_{1}=0.2, \lambda_{2}=0.5, \lambda_{3}=0.8, k_{2}=1$, and $\mu_{1}=$ 0.5 . The red mesh indicates the part of the wing surface that is modified by this parametric scheme.

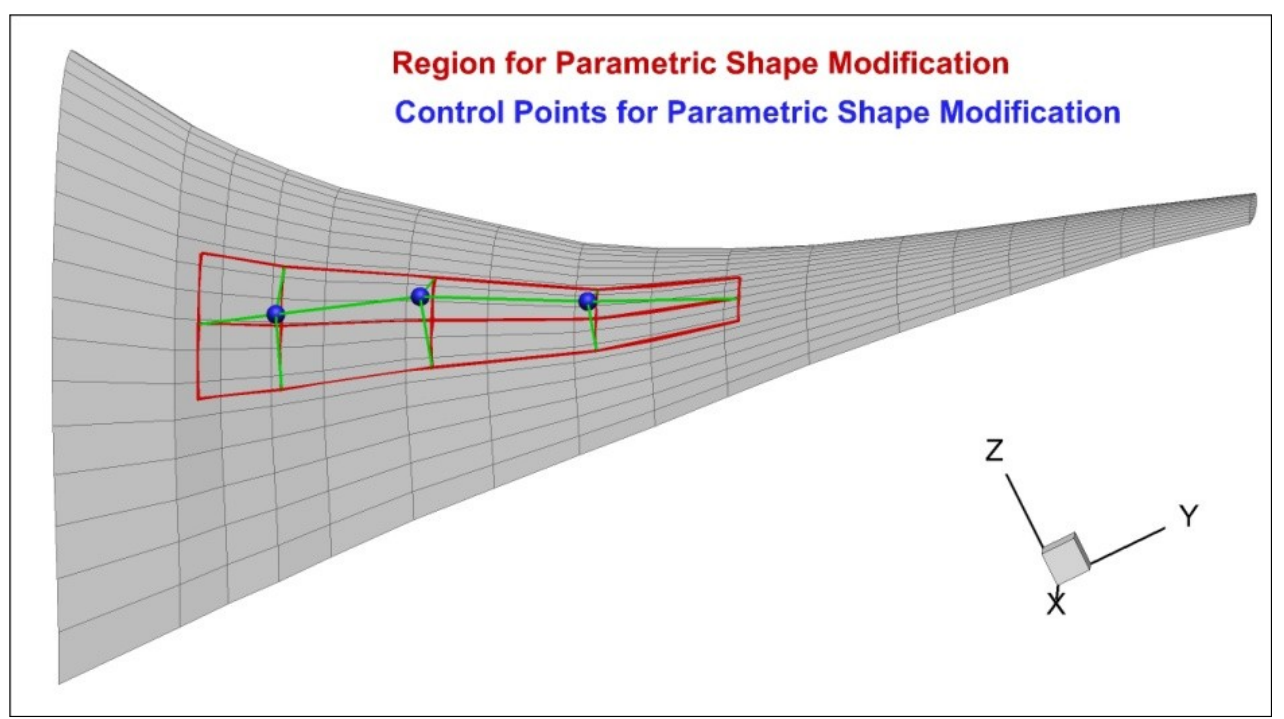

Figure 3. A parametric scheme for wing surface modification.

A user also can choose to arbitrarily change a wing-like surface. This option for the lifting-surface scheme does not require the same number of points on the upper and lower surfaces of an airfoil section, and $v_{j}$ are defined for all $j$ indices. The control surface $f(u, v)$ is defined similarly as before, and the wing surface points are modified by adding $f\left(u_{i}, v_{j}\right)$ to $z[i, j]$ for all $i$ and $j$. A more general method for changing a part of a set of discrete surface points $\left\{P_{i}: 1 \leq i \leq n\right\}$ is discussed in reference [13]. This method uses a control NURBS surface $f(u, v)$ in space to mark the modification region for the discrete points, determines the values of $u_{i}$ and $v_{i}$ for each point $P_{i}$ in the modification region, and generates the updated surface points by adding the change in $f\left(u_{i}, v_{i}\right)$ to $P_{i}$. The design variables are the control points for the NURBS surface $f(u, v)$.

The twist scheme is simply a special case for modifying the camber by using the lifting-surface scheme. Let $v_{\min }$ $=\mu_{1}=0, v_{\max }=\mu_{2}=1\left(k_{2}=2\right), \Delta Z_{i, 1}=0$ for all $i$. Then, the lifting surface scheme for camber modifications leads to a wing twist with a fixed leading edge, while a wing twist with a fixed trailing edge can be implemented with $\Delta Z_{i, 2}=$ 0 for all $i$. Because the twist scheme is a commonly used method for tailoring the lift distribution, a customized user interface is provided for convenience. Users only need to indicate whether the leading or the trailing edge is fixed and specify the values of the twist angles at the control-point locations, which are translated internally to $\Delta Z_{i, j}$ for the corresponding lifting-surface scheme.

The body-surface scheme is similar to the lifting-surface scheme for making arbitrary shape modifications. The difference is that the control points $R_{i, j}$ represent the changes in the outer normal direction of the underlying bodytype surface. The parameter $u_{i}$ represents the percentage location in the longitudinal direction for cross section $i$, and $v_{j}$ represents the approximate percentage location of the streamline $j$ in the azimuthal direction. A user has two options for defining the outer normal directions $Q_{i, j}$ : the surface normal or the radial normal. If the surface normal option is selected, then $Q_{i, j}$ is the unit outer normal vector at $\left(u_{i}, v_{j}\right)$ for the B-spline surface interpolation of the data points

$$
P[i, j]=(x[i, j], y[i, j], z[i, j]) \text { for } 1 \leq i \leq m \text { and } 1 \leq j \leq n .
$$


If the radial normal option is selected, then $Q_{i, j}$ is the unit vector scaled from $P[i, j]-W_{i}$, where $W_{i}$ is the centroid of the cross section $i$ defined by $P[i, j](1 \leq j \leq n)$. The body-surface points are modified by adding $f\left(u_{i}, v_{j}\right) \cdot Q_{i, j}$ to $P[i, j]$ for $1 \leq i \leq m$ and $1 \leq j \leq n$, where $f(u, v)$ is defined by a B-spline interpolation of the control points

$$
\left\{R_{i, j}: 1 \leq i \leq k_{1} \text { and } 1 \leq j \leq k_{2}\right\}: \quad f\left(u_{i, c}, v_{j, c}\right)=R_{i, j}
$$

In Fig. $4, u_{\min }=0.5, u_{\max }=0.7, v_{\min }=0.4, v_{\max }=0.7, k_{1}=1, \lambda_{1}=0.5, k_{2}=1$, and $\mu_{1}=0.5$. The red mesh indicates the part of the fuselage surface that is modified by this parametric scheme. The modified surface (not shown in Fig. 4) passes through the blue point and blends smoothly from the blue point to the boundary of the modification region.

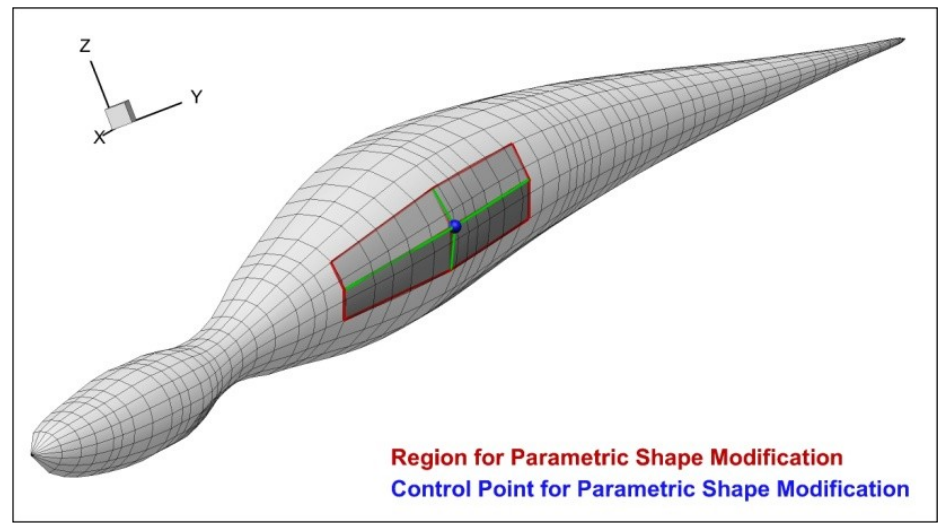

Figure 4. A parametric scheme for body-surface modification.

The body-scaling scheme can be used to scale the width distribution of the cross sections or to scale each cross section from its centroid. The control points are the scaling factors $S_{i}$ along the specified longitudinal locations $0 \leq$ $\lambda_{1}<\ldots<\lambda_{k} \leq 1$. The actual longitudinal locations are defined by $u_{i, c}=u_{\min }+\lambda_{i} \cdot\left(u_{\max }-u_{\min }\right)$. The scaling curve is a B-spline interpolation of the scaling factors $S_{i}: f\left(u_{i, c}\right)=S_{i}$ for $1 \leq i \leq k$. To scale from the centroid, the body-surface points are updated by replacing $P[i, j]$ with $W_{i}+f\left(u_{i}\right) \cdot\left(P[i, j]-W_{i}\right)$, where $W_{i}$ is the centroid of the cross section $i$ defined by $P[i, j](1 \leq j \leq n)$. For scaling by width, the body-surface points are modified by replacing $y[i, j]$ with $W_{i, y}$ $+f\left(u_{i}\right) \cdot\left(y[i, j]-W_{i, y}\right)$, where $W_{i, y}$ is the $y$ coordinate of the centroid of the cross section $i$.

The body camber-line scheme is similar to the body-scaling scheme, except that the body-surface points are modified by adding $f\left(u_{i}\right)$ to $z[i, j]$. In Fig. 5, a single design variable is used to move the fuselage camber line downward.

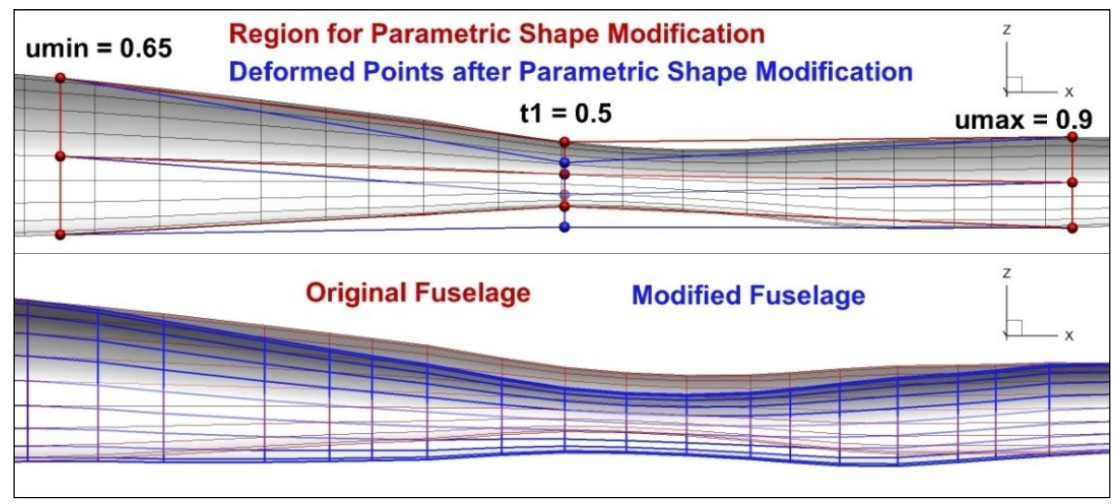

Figure 5. A parametric scheme for body camber-line modification.

The shifting and scaling scheme uses the formula

$$
x_{\text {new }}[i, j]=x_{\mathrm{W}}+x_{\mathrm{S}} \cdot\left(x[i, j]-x_{\mathrm{c}}\right)+x_{\mathrm{t}},
$$


where $x_{\mathrm{W}}$ is the $x$ coordinate of the scaling center, $x_{\mathrm{S}}$ is the $x$ coordinate of the scaling vector, and $x_{\mathrm{t}}$ is the $x$ coordinate of the translation vector. The $y$ and $z$ coordinates are updated with similar formulas.

Finally, the linear morphing scheme is the simplest application of the domain-element approach. The rectangular control box is defined to be the smallest rectangular box that contains the component to be modified. This control box has eight vertices, which are the design variables. Note that a linear morphing scheme has a maximum of 24 design variables. A user can easily add more control points between the vertices of the control box and use radial basis functions for nonlinear morphing, as demonstrated in reference [4], but the number of design variables increases drastically. Figure 6 shows how to use linear morphing to simulate a twist of the wing by modifying four $z$ coordinates out of 24 design variables.

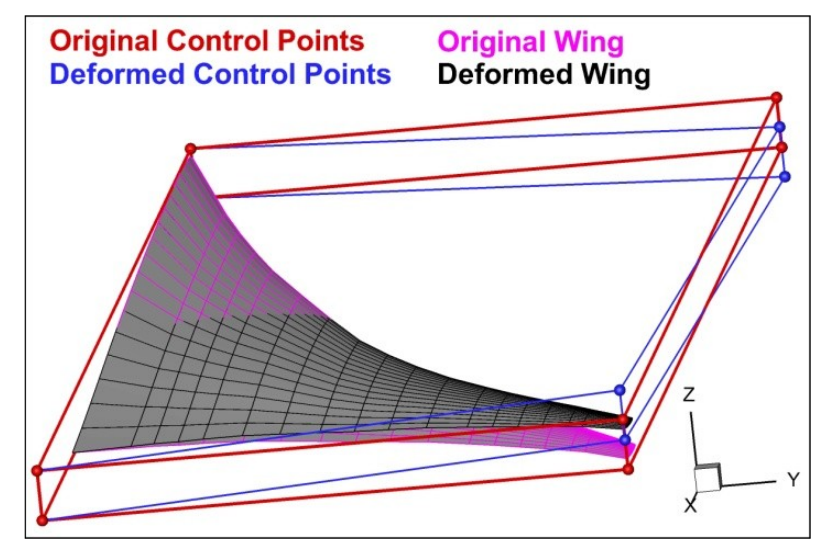

Figure 6. A parametric scheme for linear morphing of wing.

\section{Applications of Parametric Schemes}

In this section, PROTEUS is applied to modify two configurations: a low-boom demonstrator concept that was developed by Elwood Shields at NASA Langley Research Center and a low-boom supersonic transport developed by The Boeing Company. The low-boom demonstrator concept was generated with VSP [5]; the low-boom supersonic transport was defined in PLOT3D format.

Figure 7 shows an example of the lifting-surface scheme applied to the wing. Two design variables are used to change the $z$ coordinates of the leading and trailing edges of the tip airfoil. The design range in the span direction is 0.4 to 1 . A wing dihedral change for the outboard portion of the wing can be simulated by using the same negative value for both design variables.

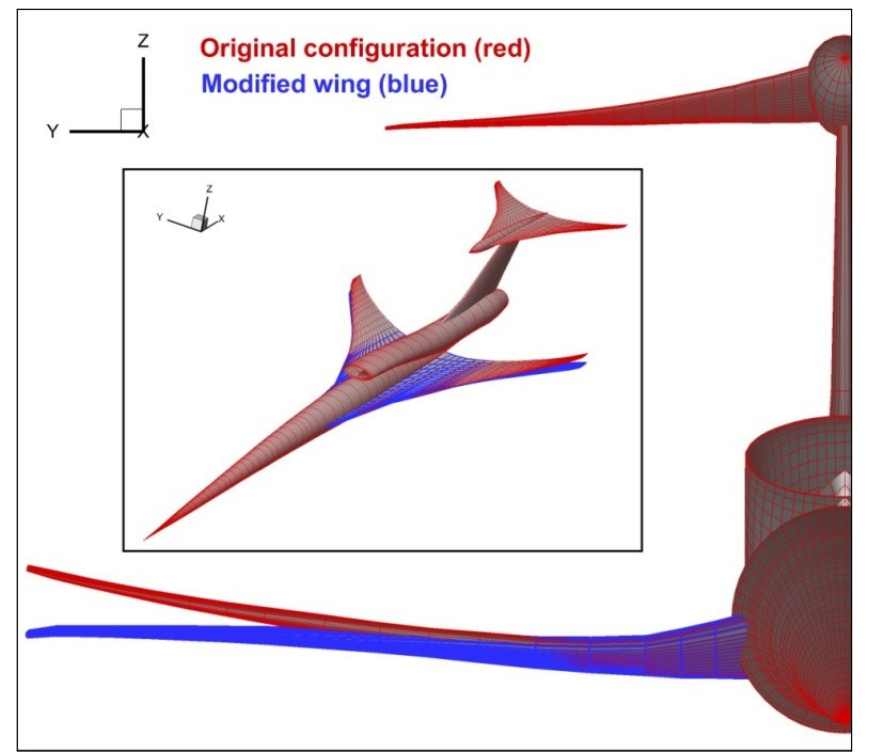

Figure 7. A camber surface scheme for wing dihedral change with two design variables.

American Institute of Aeronautics and Astronautics 
The planform scheme can be used to simulate a wing sweep modification. In Fig. 8, the design range in the span direction is 0.2 to 1 , and one negative number is used to change the $z$ coordinates of the leading and trailing edges of the tip airfoil. This leads to a sweep change from 20 percent of the span location to the tip airfoil.

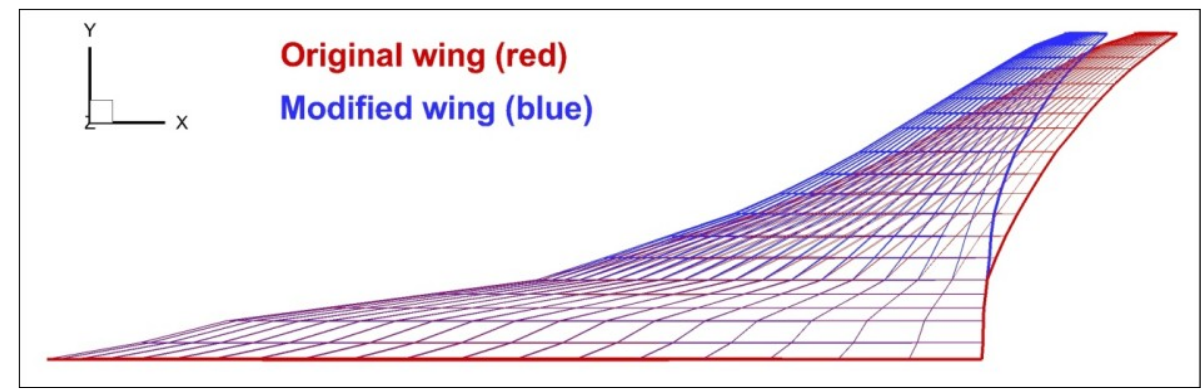

Figure 8. Wing sweep modification with two design variables.

Figures 9 and 10 illustrate a parametric scheme for modifying the camber surface of a horizontal tail. This parametric scheme was used to improve the aft signature of a low-boom supersonic demonstrator [14]. Among the nine control points that are shown in Fig. 9, the leading edge of the tip airfoil is fixed, and the middle control point at the tip airfoil equals one-half of the trailing-edge control point, which mimics a twist of the tip airfoil. In addition, the span location of the control points for the middle airfoil is used as a design variable. As a result, this parametric scheme has eight design variables. The final optimized horizontal tail of the low-boom optimization result (also see ref. [14]) is shown in Figs. 9 and 10. The airfoils are plotted with $z / x=4$ to magnify the changes in $z$. Note that no parameterization scheme in VSP can simulate the camber surface modifications that are generated by this scheme.

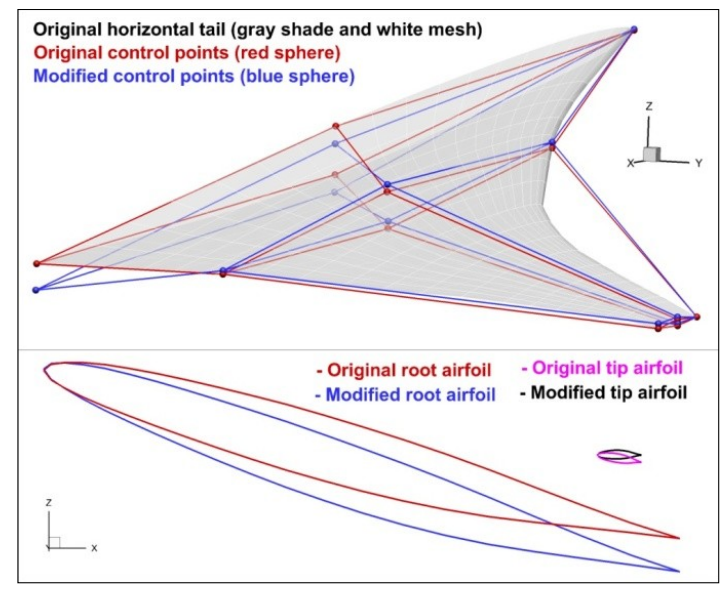

Figure 9. Horizontal-tail camber surface modification with eight design variables.

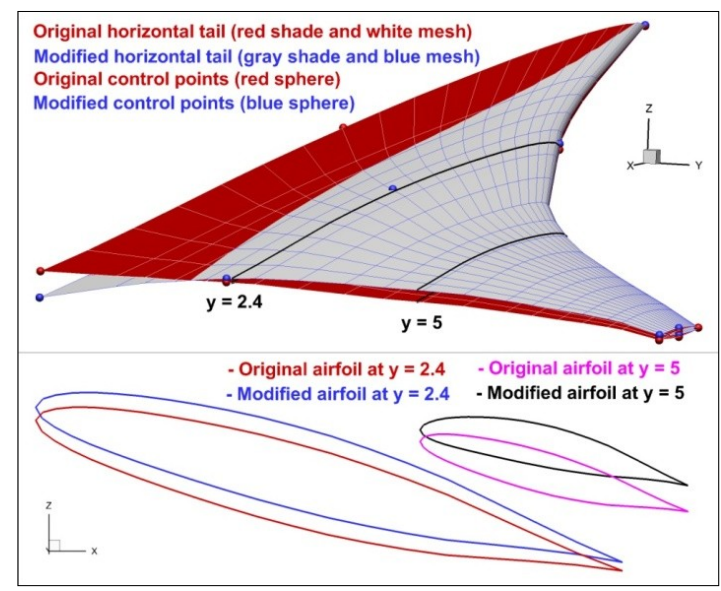

Figure 10. Comparison of surfaces and airfoils for horizontal-tail camber surface modification. 
Next, the thickness distribution of a wing is modified by using four design variables at four span locations along the mid-chord location. Here, the entire wing is modified by setting $u_{\min }=0, u_{\max }=1, v_{\min }=0$, and $v_{\max }=1$. In the span direction, the percentages of the span locations for four control points are set to $\lambda_{1}=0, \lambda_{2}=0.2, \lambda_{3}=0.6$, and $\lambda_{4}=1$. The only chord location for the control points is $\mu_{1}=0.5$. Figure 11 shows a wing that was modified by using this parametric scheme. The wing surfaces and the airfoils are plotted with $z / x=7$ to magnify the changes in $z$.

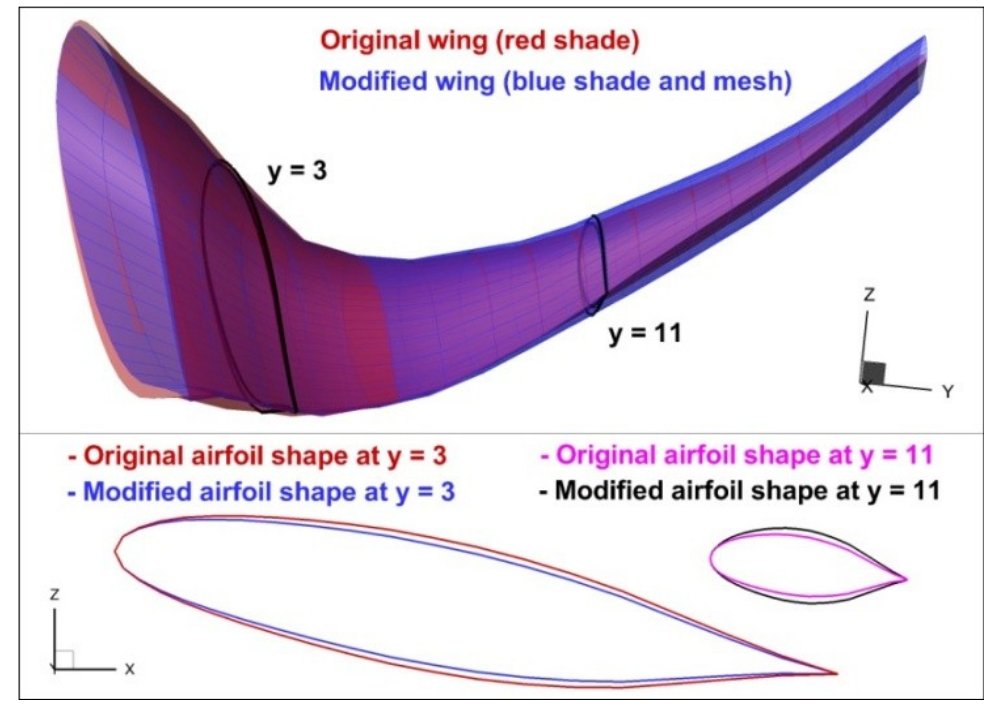

Figure 11. Wing thickness modification with four design variables.

The remaining examples are applications of PROTEUS to the low-boom supersonic transport. The first example, shown in Fig. 12, demonstrates a fuselage camber-line modification with five design variables at five equally spaced longitudinal locations.

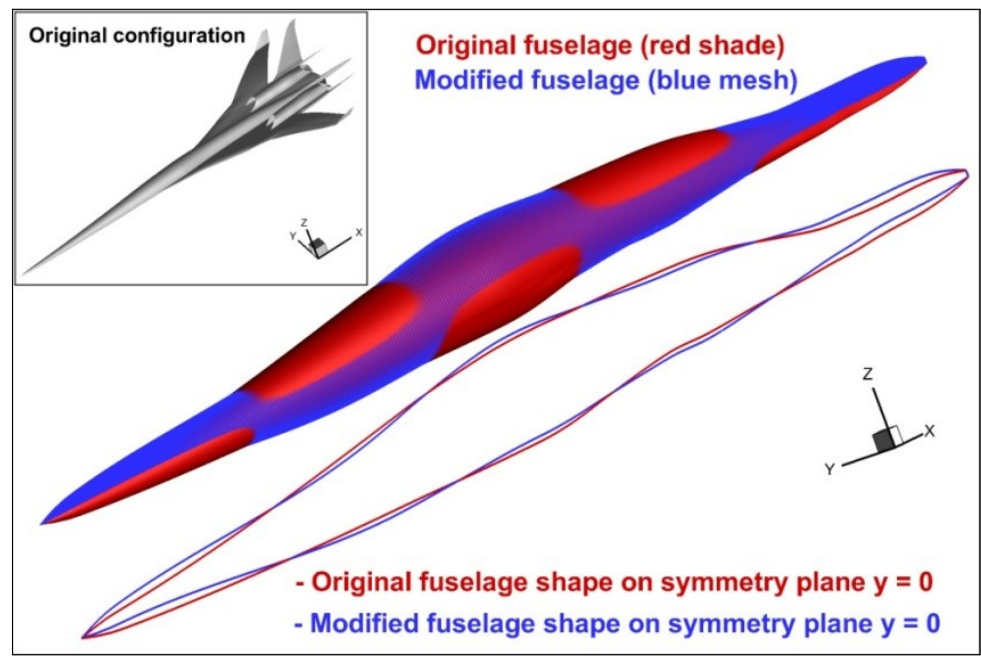

Figure 12. Fuselage camber modification with five design variables.

The next two examples illustrate two different scaling schemes for the fuselage cross sections and demonstrate the ease with which the parametric scheme can be changed in PROTEUS. The control point locations are set at $\lambda_{1}=$ $0.2, \lambda_{2}=0.4, \lambda_{3}=0.6$, and $\lambda_{4}=0.8$. By choosing $u_{\min }=0$ and $u_{\max }=1$ and selecting the width-scaling option, the entire fuselage is scaled in width with four design variables (see Fig. 13). To keep the first and the last 20 percent of the fuselage unchanged, $u_{\min }$ and $u_{\max }$ are set at 0.2 and 0.8 (instead of 0 and 1), respectively. By selecting the "Cross-section scaling from centroid" option in PROTEUS, the same set of design variables changes the fuselage quite differently, as shown in Fig. 14. These two examples demonstrate the reusability and flexibility of the parametric schemes in PROTEUS. 


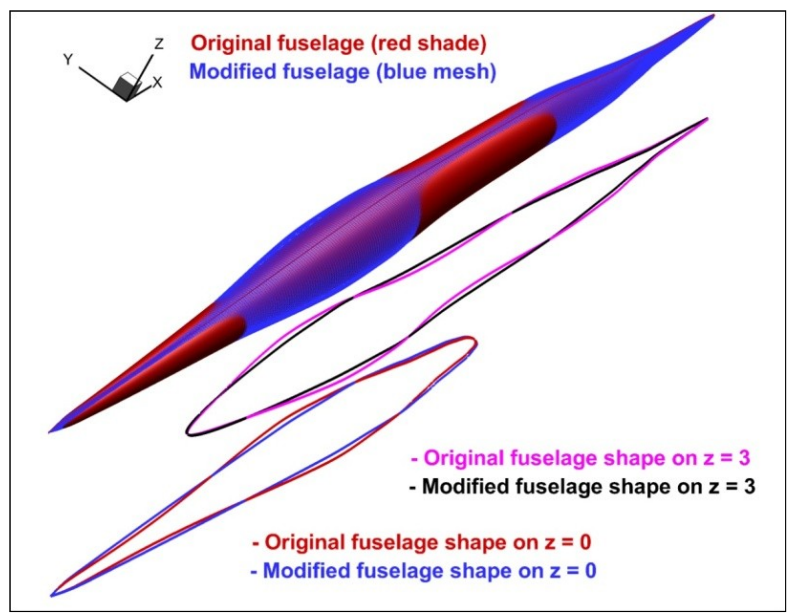

Figure 13. Fuselage width scaled with four design variables.

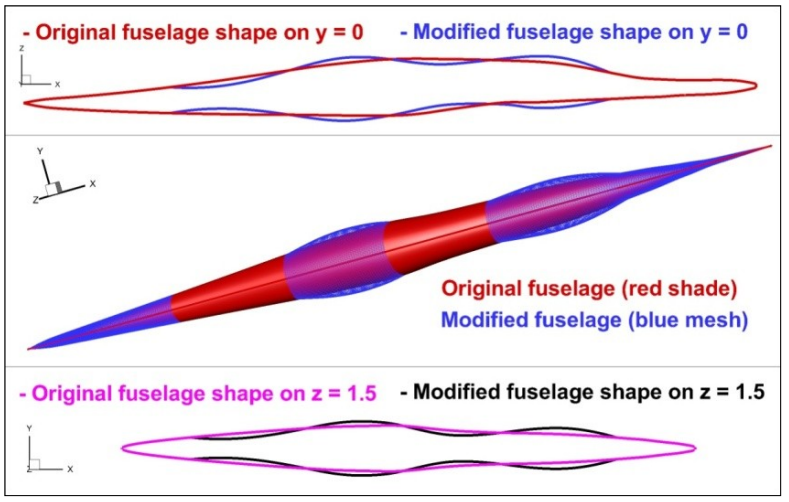

Figure 14. Fuselage scaled from centroid with four design variables.

The body-scaling scheme does not allow arbitrary changes to the cross-sectional shape and usually leads to relatively smooth changes in the body surface. The body-surface scheme can be used to make arbitrary modifications to the cross-sectional shape. In Fig. 15, the fuselage surface is modified by using six design variables in the region that is bounded by the longitudinal locations of 0.2 and 0.5 and the azimuthal locations of 0.3 and 0.7 . This shape modification may not be desirable but is intended to demonstrate the flexibility of the body-surface scheme in making cross-sectional changes.

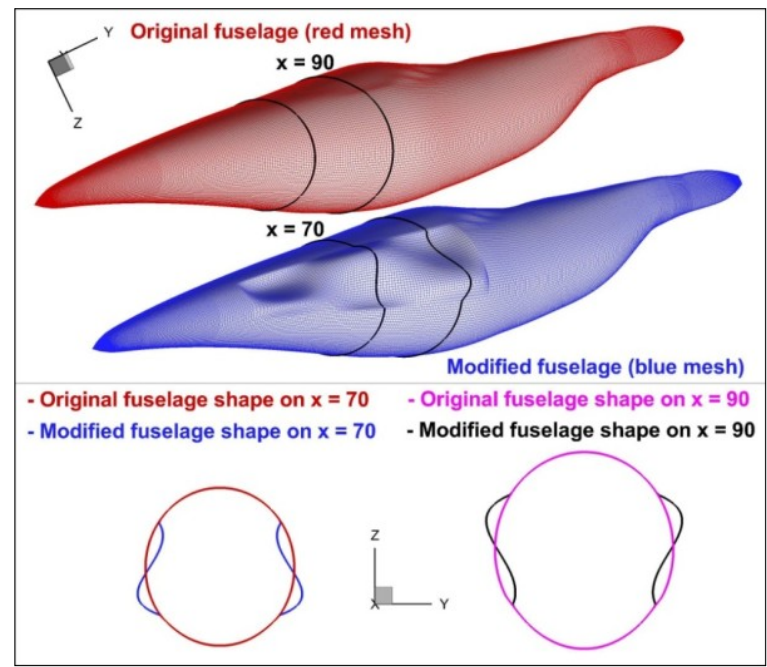

Figure 15. Aribitrary fuselage surface modification with six design variables.

10

American Institute of Aeronautics and Astronautics 
The wing-type surfaces of the low-boom supersonic transport do not satisfy the data-structure requirement of PROTEUS for wing-type surfaces (i.e., the upper and lower surfaces have the same number of points for each airfoil station). Therefore, the planform scheme and the camber/thickness scheme in PROTEUS cannot be applied directly to this configuration; however, the arbitrary surface modification option of the lifting-surface scheme can be applied. Figure 16 shows the modification of one side of the vertical tail with four design variables. Again, this example is intended only to demonstrate the flexibility of the parametric scheme for changing wing surface shapes.

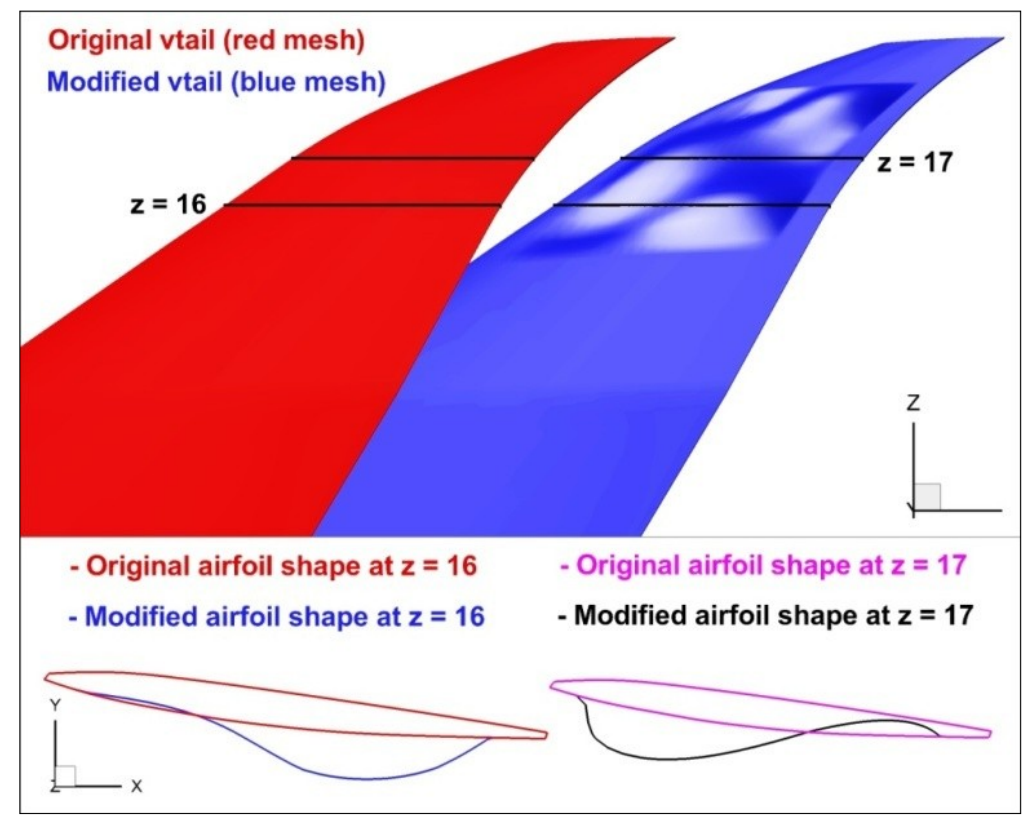

Figure 16. Arbitrary vertical-tail surface modification with four design variables.

A twist for the vertical tail can be simulated by using two design variables and the arbitrary shape-modification option for the lifting-surface scheme. In this case, $u_{\min }=0.8, u_{\max }=0.9, v_{\min }=0$, and $v_{\max }=1$ (i.e., the part of the vertical tail that is close to the tip airfoil is modified). The only span location for the control points is $\lambda_{1}=0.5$. For the chord direction, $k_{2}=2, \mu_{1}=0$, and $\mu_{2}=1$, which correspond to the two trailing-edge points on the upper and lower surfaces. Figure 17 compares the original and modified vertical tails with two design variables at the same positive value.

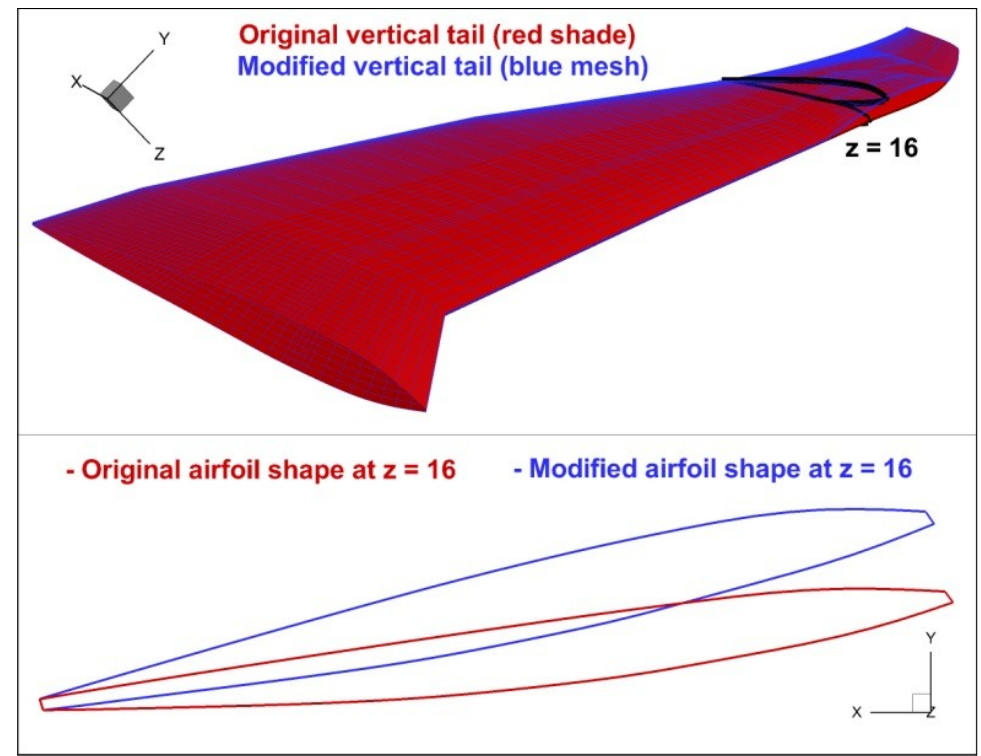

Figure 17. Vertical tail twisted around the trailing edge by using two design variables.

11

American Institute of Aeronautics and Astronautics 
Similarly, modifications to the wing camber surface can be implemented by using two arbitrary surface modification schemes: one for the upper surface and another for the lower surface. For this example, the span range is $u_{\min }=0.1$ to $u_{\max }=0.5$, and the span location parameters are $\lambda_{1}=0.3, \lambda_{2}=0.6$, and $\lambda_{3}=0.8$. The chord locations for the first scheme range from $v_{\min }=0.01$ to $v_{\max }=0.08$, and the chord locations for the second scheme range from $v_{\min }=0.92$ to $v_{\max }=0.99$. The chord design-variable location for both schemes is 0.5 . If the same value is used for the two control points for these two schemes at the same span location, then the simulated camber surface shape modifications can be achieved (see Fig. 18).

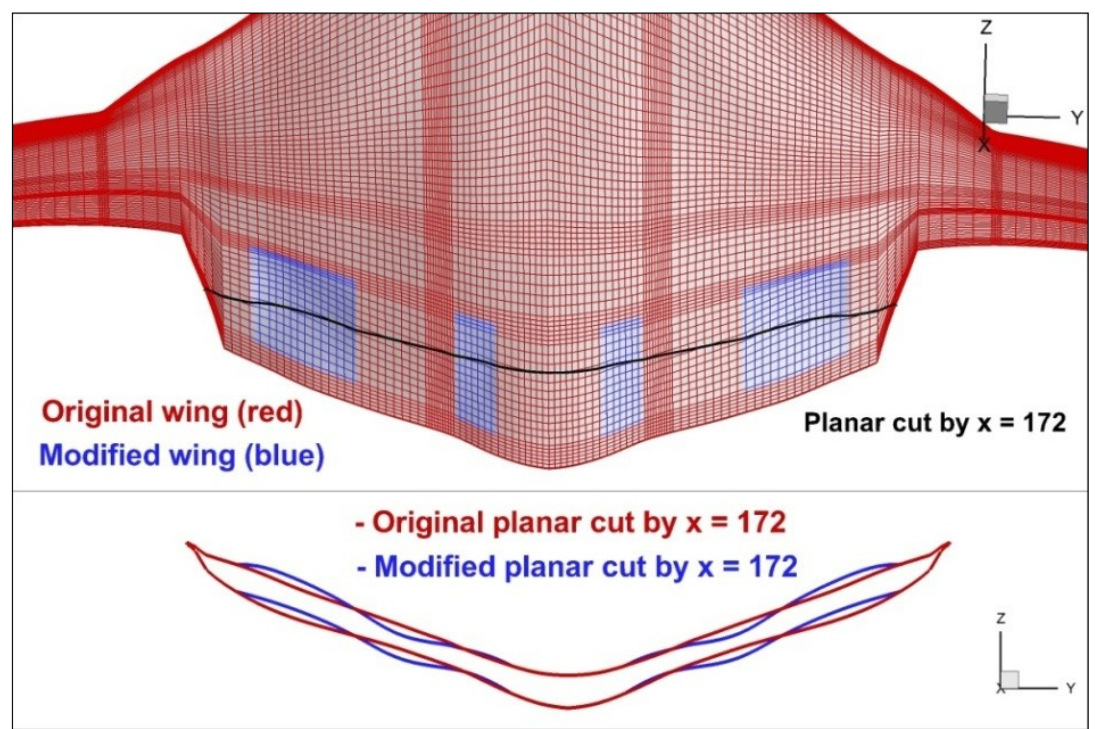

Figure 18. Wing camber surface modification with three design variables.

\section{Concluding Remarks}

A rapid shape parameterization tool called PROTEUS is developed for aircraft shape optimization. This tool can be applied directly to any aircraft geometry that has been defined in PLOT3D format. The only restriction is that only wing-type (i.e., defined by airfoil station) and body-type (i.e., defined by cross section) surfaces can be modified with PROTEUS. The eight parametric schemes in PROTEUS allow users to quickly set up (i.e., in just a few minutes) a parametric scheme by specifying the design ranges and the locations of the design variables. The design ranges and design-variable locations are independent of the underlying surface; this feature allows the application of a specific parametric scheme to any surface of the same type.

Available parametric geometry tools can be classified as either parametric shape modification tools (e.g., SCULPTOR [3]), which can be used for computational-fluid-dynamics (CFD) geometry shape optimization, and parametric shape construction tools (e.g., Vehicle Sketch Pad (VSP) [5]), which are mainly for the initial layout and optimization of aircraft components. Shape modification tools are applicable to any geometry but lack the capability for exploiting the features of aircraft wing-type and body-type surfaces. Parametric shape construction tools can take advantage of wing-type and body-type surface structures for the easy construction of components; however, the application of new parametric schemes to existing configurations can be cumbersome. PROTEUS fills a gap between current shape modification and shape construction tools; PROTEUS simplifies the setup process for different parametric schemes for existing aircraft components. The examples in this paper demonstrate that commonly used surface shape modification schemes (e.g., twist, dihedral change, sweep change, camber and thickness change, and cross-sectional scaling) can easily be created with PROTEUS.

\section{Acknowledgments}

The author would like to thank Dr. Irian Ordaz and Dr. Sriram Rallabhandi for implementing the shape parameterization tool PROTEUS in ModelCenter. 


\section{References}

[1] Samareh, J., "Survey of Shape Parameterization Techniques for High-Fidelity Multidisciplinary Shape Optimization," AIAA Journal, Vol. 39, No. 5, 2001, pp. 877-884.

[2] Samareh, J., "Novel Multidisciplinary Shape Parameterization Scheme," Journal of Aircraft, Vol. 38, No. 6, 2001, pp. 1015-1024.

[3] "SCULPTOR: Solutions Overview," Optimal Solutions Software, URL: http://www.optimalsolutions.us/solutions, accessed October 2011.

[4] Morris, A., Allen C., and Rendall, T., "Domain Element Method for Aerodynamic Shape Optimization Applied to Modern Transport Wing," Journal of Aircraft, Vol. 47, No. 7, 2009, pp. 1147-1659.

[5] Hahn, A., "Vehicle Sketch Pad: A Parametric Geometry Modeler for Conceptual Aircraft Design," AIAA Paper 2010-657, January 2010.

[6] Kulfan, B., "Universal Parametric Geometry Representation Method," Journal of Aircraft, Vol. 45, No. 1, 2008, pp. 142-158.

[7] Ordaz, I., "Conversion of Component-Based Point Definition to VSP Model and Higher Order Meshing," AIAA Paper 2011-358, January 2011.

[8] Li, W., Shields, E., and Geiselhart, K., "Mixed-Fidelity Approach for Design of Low-Boom Supersonic Aircraft," AIAA Paper 2010-0845, January 2010. Also in Journal of Aircraft, Vol. 48, No. 4, 2011, pp. $1131-1135$.

[9] Ordaz, I. and Li, W., "Integration of Off-Track Sonic Boom Analysis in Conceptual Design of Supersonic Aircraft," AIAA Paper 2011-464, January 2011.

[10] Ordaz, I., "Boom Minimization Framework for Supersonic Aircraft Using CFD Analysis," AIAA Paper 20101506, January 2010.

[11] "ModelCenter and Optimization Tools," Phoenix Integration, URL: http://www.phoenix-int.com, accessed May 2011.

[12] “TECPLOT 360: User's Manual,” Tecplot, Inc., URL: http://www.tecplot.com/Support/Documentation.aspx, accessed October 2011.

[13] Samareh, J., "Aerodynamic Shape Optimization Based on Free-form Deformation," AIAA Paper 2004-4630, September 2004.

[14] Ordaz, I. and Li, W., "Adaptive Aft Signature Shaping of a Low-Boom Supersonic Aircraft Using Off-Body Pressures," AIAA Paper 2012-XXX, The 50th AIAA Aerospace Sciences Meeting, Nashville, Tennessee, January 2012. 